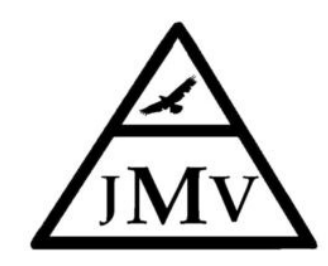

\title{
Patient Information
}

Series 2

\section{Oxygen support systems}

in the last series, we talked about various reasons why can our respiratory system fails. The symptoms usually include but not limited to shortness of breath, cough, increasing sputum production, inability to exercise or difficult ambulating.

Most of those diseases can alter the levels of oxygen (Hypoxia) and carbon dioxide (Hypercarbia) in our body causing further deterioration in the body functions.

The level of oxygen support delivered in these cases will depend on the disease, its severity and most importantly on the patient condition. We will review some of those devices with brief explanation of each.

A) Nasal cannula: is the simplest form of oxygen delivery method which supplies low flow oxygen in the range of $0.5-8$ Liters per minute which usually translates to $22-40 \%$ fractional inspired oxygen known as $\mathrm{FiO} 2$ (normal air has $21 \% \mathrm{FiO} 2$ ). This is used in mild-moderate cases of hypoxia. Its advantage is: its cheap, widely available, can be used at home, but its advantages are failure in severe cases, easily dislodged, dries the nose, can be uncomfortable, less effective if patient is mouth breather.

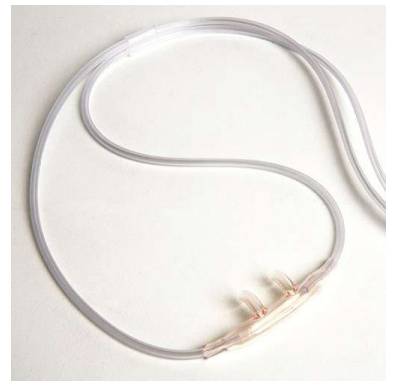

B) Simple face mask: this mask delivers slightly higher flow: 5-10 liters per minute and slightly higher oxygen content $35-50 \%$ compared to nasal cannula. Its advantages are cheap, available, but its disadvantages are it can be uncomfortable, difficult to eat with it. 


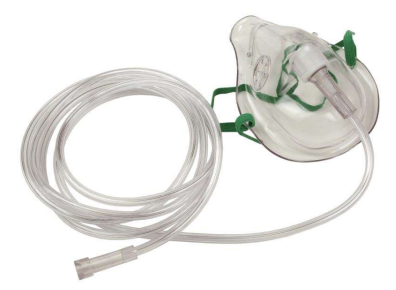

C) Partial rebreathing mask: those masks have a 1 lit bag attached to the mask that acts as a reservoir of oxygen and would be inflated all time. It provides slightly higher flow of 10-15 liters per min with higher oxygen content of $40-70 \%$. This mask has no valves on it. Those are used in higher degree of hypoxia. Advantages are higher oxygen delivery than simple mask, disadvantages are similar.

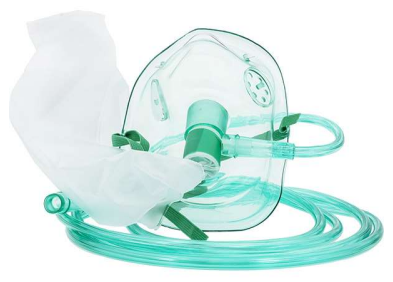

D) Non rebreathing masks: those look similar to the partial rebreathing masks but have valves on them so expired air does not go to the reservoir bag. It provides flow about 10-15 liters per minute but slightly higher oxygen at $60-80 \%$. Advantages and disadvantages as above mask.

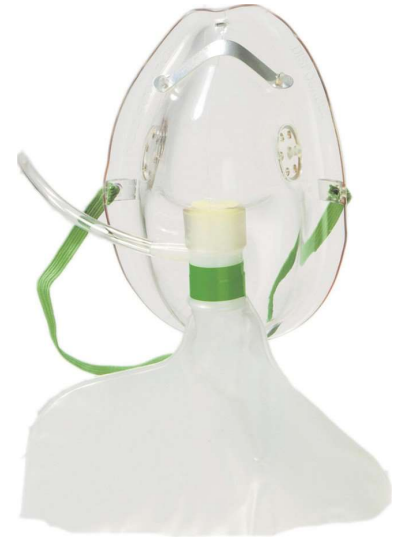

E) High flow nasal cannula: those are sophisticated devices that has recently gained much momentum in the medical field. They supply heated humidified oxygen at a remarkably high flow rate up to 60-70 liters per minute and oxygen concentration up to $100 \%$. Their advantages are they are comfortable, decreases air hunger but disadvantages are some patients can not tolerate very high flow of air, they are not widely available, need expertise and may need to be monitored in intensive care unit or high level monitoring.

F) Noninvasive positive pressure: commonly referred to as BIPAP, those are basically a form of mechanical ventilators that delivers the oxygen at very high flow and concentrations up to $100 \%$ 
but through different full tight face masks, nasal masks, prongs, or head helmets. The term noninvasive indicates that we do not use an artificial airway (endotracheal tube or tracheostomy tube). Those are commonly used for cases of heart failure, COPD or post operatively though they can be used in other forms of diseases especially the ones that result in high carbon dioxide.
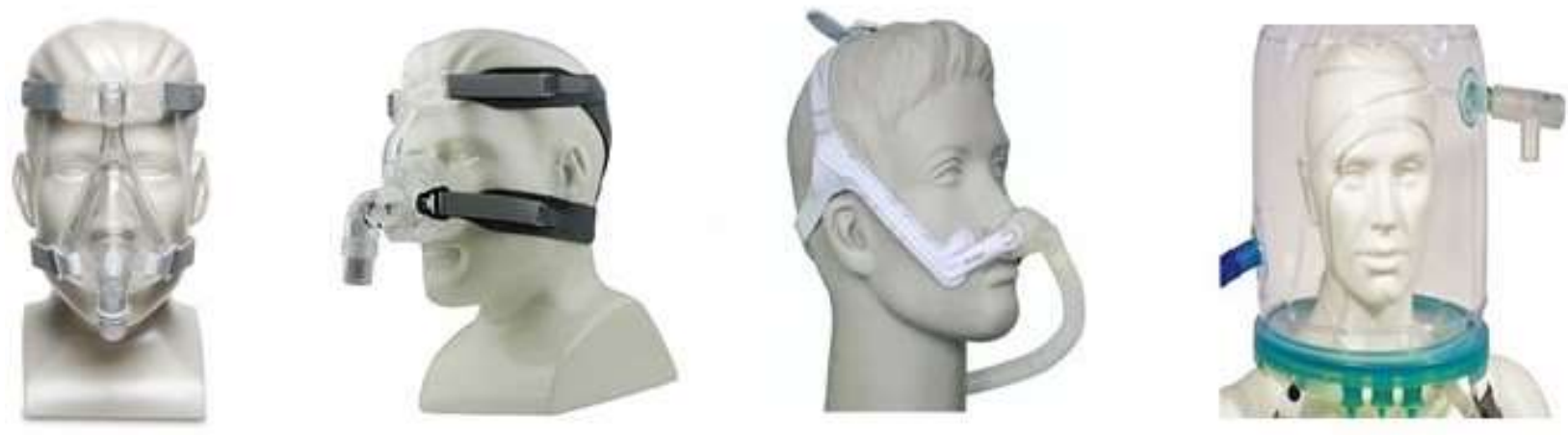

G) Invasive mechanical ventilation: This is the most sophisticated form of respiratory support and we will discuss it in a future series. Those machines operate by supplying high flow and high oxygen through high pressures applied directly to the lungs through an artificial airway as mentioned above. Patients on those machines have to be cared in intensive care units, though stable patients with chronic respiratory failure can use those machines in nursing homes or homes.
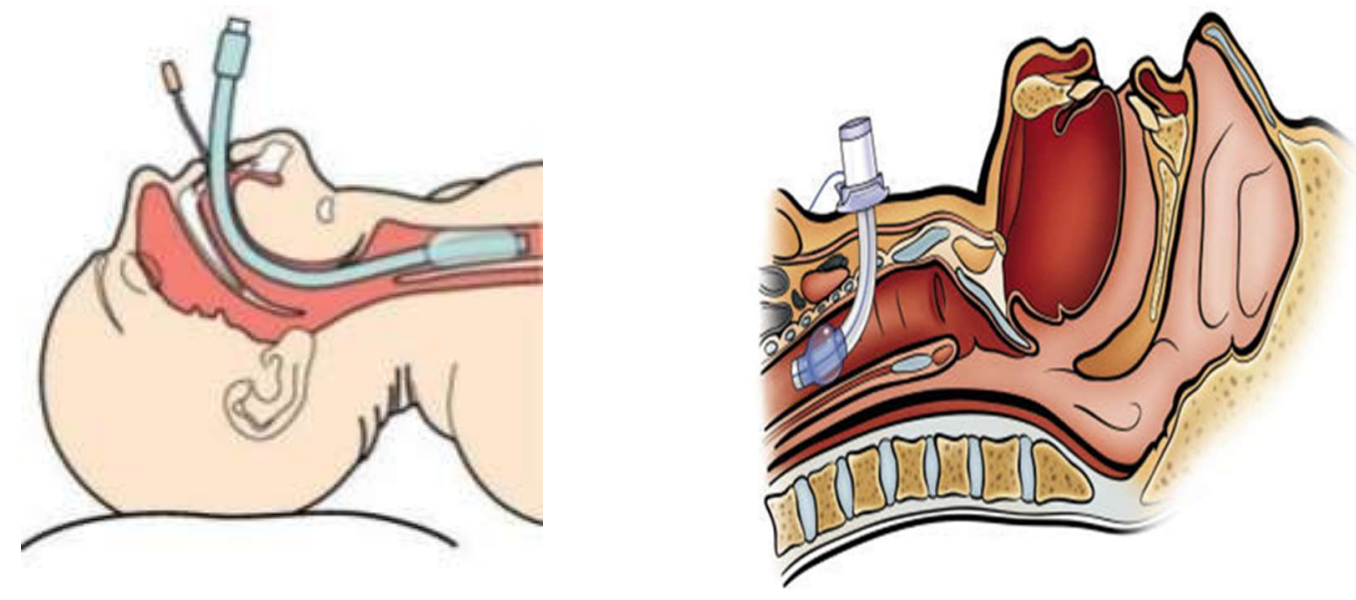

Endotracheal tube going through the mouth that connects ventilator to the lungs
Tracheostomy tube going through the neck that connects ventilator to the lungs 


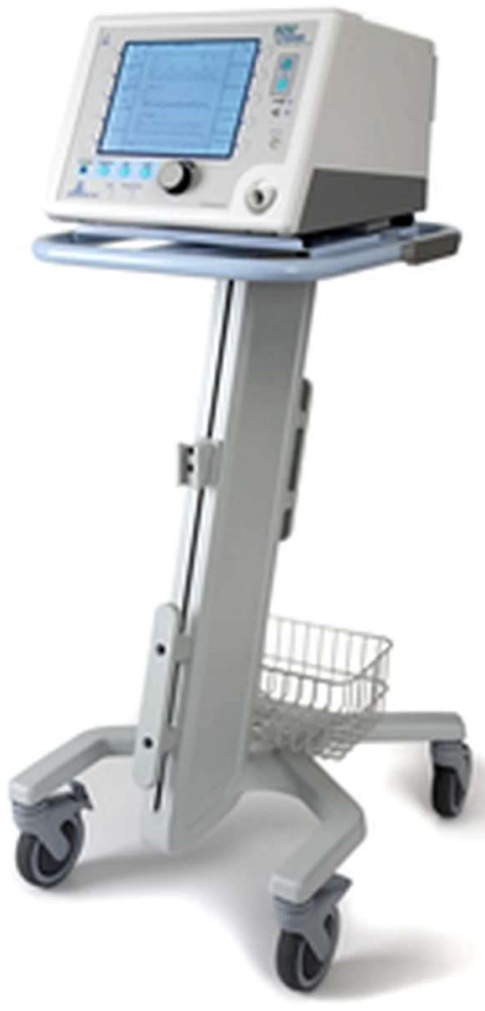

Sample of the noninvasive ventilators

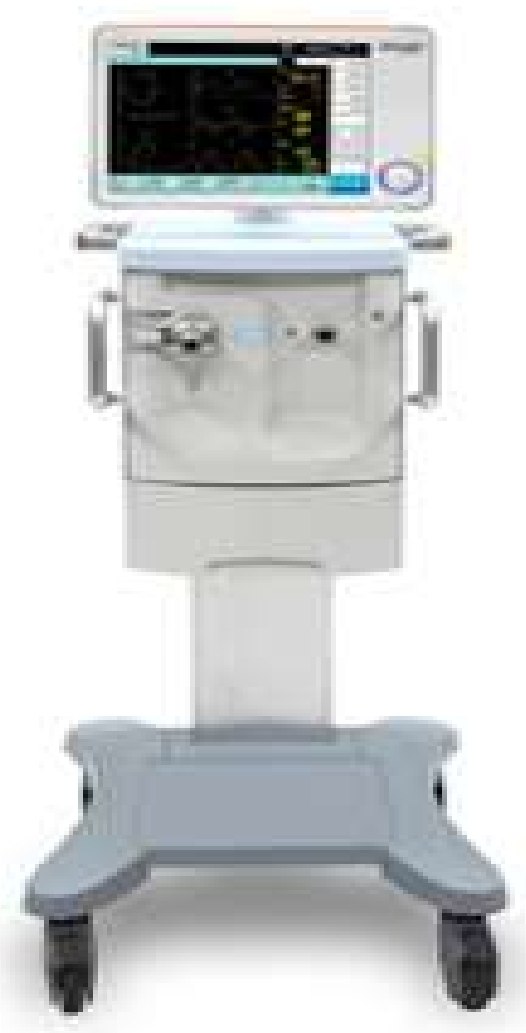

